\title{
Effect of Rotation on Scaffold Motion and Cell Growth in Rotating Bioreactors
}

\author{
Mark C. Varley, PhD, ${ }^{1}$ Athina E. Markaki, PhD, and Roger A. Brooks, $\mathrm{PhD}^{2}$
}

Efficient use of different bioreactor designs to improve cell growth in three-dimensional scaffolds requires an understanding of their mechanism of action. To address this for rotating wall vessel bioreactors, fluid and scaffold motion were investigated experimentally at different rotation speeds and vessel fill volumes. Low cost bioreactors with single and dual axis rotation were developed to investigate the effect of these systems on human osteoblast proliferation in free floating and constrained collagen-glycosaminoglycan porous scaffolds. A range of scaffold motions (free fall, periodic oscillation, and orbital motion) were observed at the rotation speeds and vessel fluid/air ratios used, with $85 \%$ fluid fill and an outer vessel wall velocity of $\sim 14 \mathrm{~mm} \mathrm{~s}^{-1}$ producing a scaffold in a free fall state. The cell proliferation results showed that after 14 and 21 days of culture, this combination of fluid fill and speed of rotation produced significantly greater cell numbers in the scaffolds than when lower or higher rotation speeds $(p<0.002)$ or when the chamber was $60 \%$ or $100 \%$ full $(p<0.01)$. The fluid flow and scaffold motion experiments show that biaxial rotation would not improve the mass transfer of medium into the scaffold as the second axis of rotation can only transition the scaffold toward oscillatory or orbital motion and, hence, reduce mass transport to the scaffold. The cell culture results confirmed that there was no benefit to the second axis of rotation with no significant difference in cell proliferation either when the scaffolds were free floating or constrained $(p>0.05)$.

Keywords: bioreactor, rotating wall vessel, biaxial, collagen scaffold, fluid flow, cell proliferation

\section{Introduction}

$\mathbf{E}$ VERY YEAR MORE THAN 1.5 million grafts are required for bone injuries ${ }^{1}$ and it is anticipated that the market for bone repair in the United States alone will be worth $\$ 3.5$ billion by $2017 .^{2}$ Tissue engineering offers the production of cellular graft substitutes as promising alternatives to traditional bone graft materials. One of the major limitations to a successful tissue engineered approach is a difficulty with scalability. In static culture, the maximum diameter of a cell spheroid is $\sim 1 \mathrm{~mm}$, beyond which a necrotic core surrounded by healthy cell forms and tissue engineering constructs is similarly limited by the ability to supply oxygen and nutrients and remove the products of cell metabolism. ${ }^{3}$ To overcome this, the cell/scaffold construct can be grown in a bioreactor, which increases the mass transfer of nutrients between the cells seeded within the scaffolds and the culture media allowing larger constructs to be produced.
One commonly used type of bioreactor is the rotating wall vessel (RWV) bioreactor, which exists in a variety of different forms. They generally consist of a circular culture vessel that rotates about its central axis. The scaffold is suspended within the fluid-filled vessel, and the angular velocity of the bioreactor is tailored to leave the scaffold in a state of "free fall." Under free fall, the scaffold appears in a fixed position within the vessel as viewed by an external observer and experiences dynamic laminar flow of culture media past and through the scaffold. ${ }^{2}$

Rotating wall bioreactors have been used to culture a large variety of cells and have been shown to improve cell proliferation by up to five times compared to static culture in a number of different scaffolds. ${ }^{4-8}$ In addition, the cells are found to be more evenly distributed throughout the scaffold. The effects of culture in RWV on osteogenic differentiation appear more variable. Some groups showed increased expression of the early stage osteogenic marker alkaline

\footnotetext{
${ }^{1}$ Department of Engineering, Cambridge University, Cambridge, United Kingdom.

${ }^{2}$ Division of Trauma and Orthopaedic Surgery, Cambridge University, Addenbrooke's Hospital, Cambridge, United Kingdom.

(C) Mark C. Varley et al. 2017; Published by Mary Ann Liebert, Inc. This is an Open Access article distributed under the terms of the Creative Commons Attribution License, which permits unrestricted use, distribution, and reproduction in any medium, provided the original work is properly cited. Mary Ann Liebert, Inc. offers reprint services for those who want to order professionally produced copies of articles published under the Creative Commons Attribution (CC BY) license. To obtain a price quote, email Reprints@ liebertpub.com. Please include the article's title or DOI, quantity, and delivery destination in your email.
} 
phosphatase, ${ }^{7,9}$ while others reported no significant increase ${ }^{4,5}$ and some have reported that RWV culture caused significant mineralization of the scaffold. ${ }^{5,8} \mathrm{~A}$ variation of the RWV bioreactor is the biaxial rotating bioreactor. ${ }^{10}$ It uses a spherical reaction vessel with the scaffolds constrained by mounting on attached needles and is able to rotate about two orthogonal axes nominally called spin and tumble with continuous medium replacement from an external reservoir. ${ }^{10}$ The biaxial rotating bioreactor was shown to improve the culture of human fetal mesenchymal stromal cells (hfMSCs) in constrained scaffolds compared to static culture ${ }^{10}$ with increased proliferation and osteogenic differentiation. In this study, hfMSCs grown on a polycaprolactone/tricalcium phosphate composite scaffold in the biaxial bioreactor reached confluence earlier, exhibited a more even tissue distribution, and showed increased mineralization compared to culture in optimized spinner flask, RWV, or perfusion bioreactors. ${ }^{11}$ The benefits in cell response observed in the biaxial rotating bioreactor were attributed to increased velocities (a factor of 10) and shear forces (a factor of 4) within the scaffolds based on previous work using computational fluid dynamics. ${ }^{12}$ However, it is not clear why the combination of two axes of rotation results in such a significant increase. Furthermore, in this study, ${ }^{12}$ both axes were rotated at $35 \mathrm{rpm}$, which is much greater than the $5 \mathrm{rpm}$ used in the cell culture experiments ${ }^{10,11}$ and gives a much higher Reynolds number and hence more turbulent flow.

The aim of this work was to understand the influence of dual axis rotation on fluid dynamics and the growth of cells when cultured in porous scaffolds. Low-cost single and dual axis rotating bioreactors were designed and developed to allow fluid volume, scaffold mounting, and bioreactor rotation speed to be optimized, and single and dual axis rotation to be compared. Fluid flow and scaffold motion were investigated experimentally for varying ratios of fluid to air. The effect of rotation speed on the scaffold flow path has been previously described for scaffolds rotating in $100 \%$ full culture vessels,,${ }^{13,14}$ but not when the fluid ratio is reduced. The effects of motion on mass transport and cell proliferation were investigated.

\section{Materials and Methods}

\section{Bioreactor design}

Two custom-built rotating bioreactors were developed, a single axis rotating bioreactor and a dual axis rotating bioreactor (Fig. 1). They were designed to take up little space, to be easily handled, and manipulated ensuring sterility. The bioreactors are powered using stepper motors controlled using an Arduino Uno microcontroller. They are primarily constructed of three-dimensional printed components made of poly-lactic acid. Both bioreactors can hold up to four reaction vessels mounted off-axis, which are secured using a friction fit. The dual axis bioreactor has an additional second axis of rotation, which is driven using a pulley, and the electronics are passed through the rotating joint using a slip ring (Fig. 1b). The single axis bioreactor can rotate at up to $50 \mathrm{rpm}$ and the dual axis bioreactor at up to $35 \mathrm{rpm}$. Both bioreactors allowed positional control to within $1.8^{\circ}$ throughout operation.

Cell culture reaction vessels were developed to undertake gas exchange and maintain sterility of culture medium. The reaction vessel was constructed from a $50 \mathrm{~mL}$ disposable cell culture tube, which had the lid drilled out. A gas permeable membrane was produced using polydimethylsiloxane, which was cast around the drilled reaction vessel lid and cured at $80^{\circ} \mathrm{C}$ for $24 \mathrm{~h}$. The adapted lids were sterilized in $70 \%$ ethanol and under ultraviolet (UV) light overnight. They were then washed in sterile phosphate-buffered saline (PBS) and dried under sterile conditions before being used on new sterile $50 \mathrm{~mL}$ cell culture tubes.

\section{Freeze-dried collagen-glycosaminoglycan scaffolds}

Collagen-glycosaminoglycan (CG) scaffolds were produced as previously described. ${ }^{15}$ All chemicals were obtained from Sigma-Aldrich. Insoluble Type I collagen $(0.5 \mathrm{wt} . \%)$ and chondroitin sulfate salts $(0.05 \mathrm{wt} . \%)$ were suspended in $0.05 \mathrm{M}$ acetic acid homogenized at $15,000 \mathrm{rpm}$ using a T81 UltraTurrax homogenizer (IKA, Germany). The suspension was poured into a polycarbonate mould and freeze dried (VirTis advantage, SP Industries, United States), cooled to $-40^{\circ} \mathrm{C}$ at a rate of $0.9^{\circ} \mathrm{C} \mathrm{min}$, and held for $60 \mathrm{~min}$ before being sublimated under a vacuum of $50 \mathrm{mT}$ Torr by raising the temperature to $0^{\circ} \mathrm{C}$ for $17 \mathrm{~h}$. Subsequently, the scaffold was cross-linked for $4 \mathrm{~h}$ using 1 ethyl-3-3-dimethyl aminopropyl carbodiimide (EDAC) with the catalyst $\mathrm{N}$-hydroxysuccinimide (NHS) at a molar ratio of $2.5 \mathrm{M}$ EDAC/M NHS. The cross-linking solution had a concentration of $6 \mathrm{mM}$ EDAC per gram of collagen.

The scaffolds produced had a relative density of 0.005 (porosity content $\sim 99.5 \%$ ) assuming a value of $1.3 \mathrm{Mg} \mathrm{m}^{-3}$ for solid collagen. ${ }_{15}^{15}$ Hydrated measurements gave a pore size of $\sim 145 \pm 70 \mu \mathrm{m} .{ }^{15}$ Cylindrical scaffolds of $5 \mathrm{~mm}$ diameter and $4 \mathrm{~mm}$ height were used in subsequent experiments.

\section{Fluid flow and scaffold tracking}

Fluid and scaffold motion within the rotating bioreactors was investigated when the axis of rotation was either perpendicular or parallel to gravity, using a customized rotation
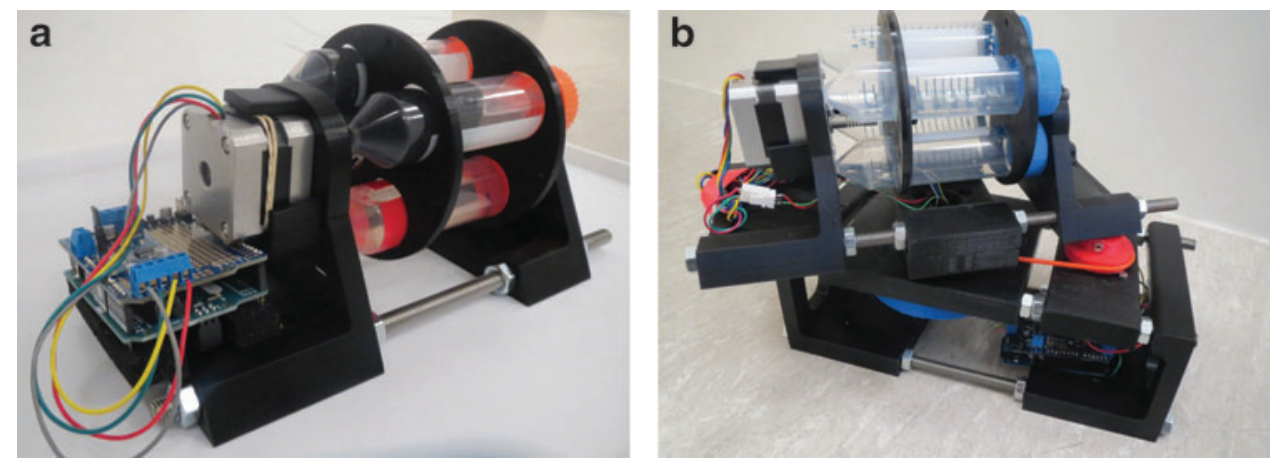

FIG. 1. Photographs of (a) single axis and (b) dual axis bioreactors. 
FIG. 2. Schematic of the setup used for measurement of the fluid flow field within a rotating bioreactor. The schematic in this study shows the setup used when the axis of rotation is orthogonal to gravity.

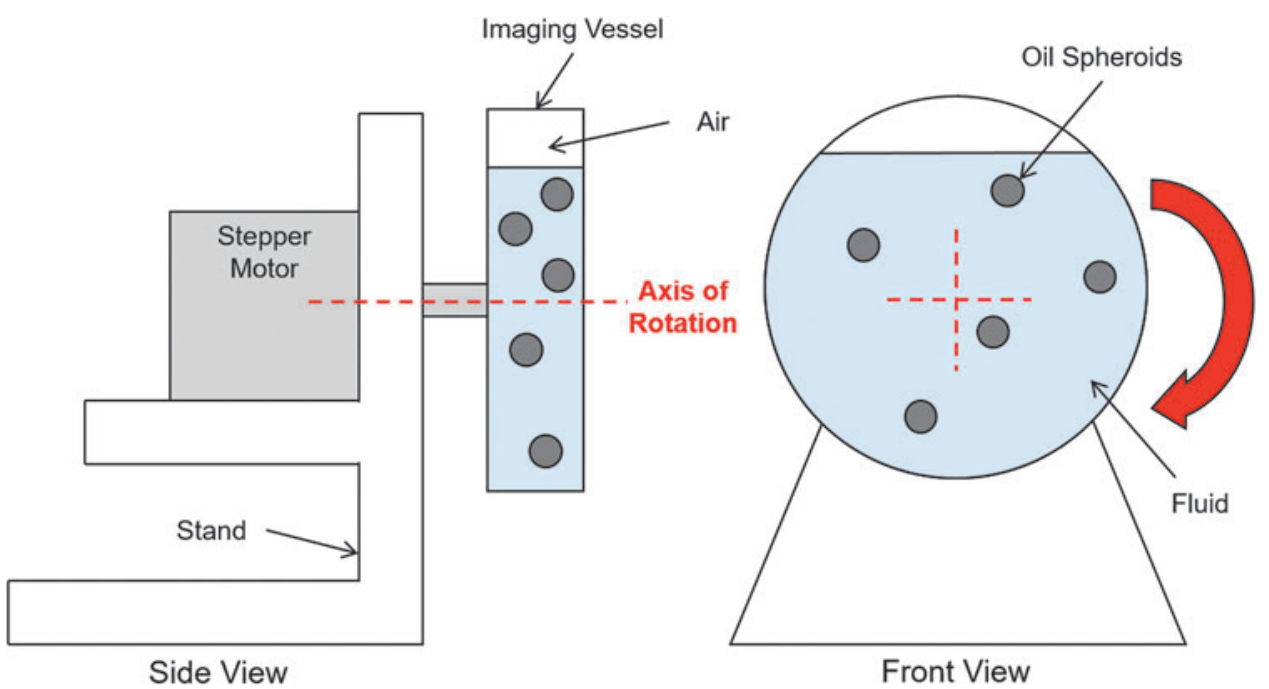

Front View rig shown schematically in Figure 2. The rig consists of a stepper motor controlled using an Arduino microcontroller, to which a $55 \mathrm{~mm}$ diameter clear cylindrical container (imaging vessel) is attached to allow fluid flow and scaffold movement to be observed.

For fluid flow analysis, the fluid inside the container consisted of a mixture of ethanol (density $0.789 \mathrm{Mg} \mathrm{m}^{-3}$ ) and water (density $0.998 \mathrm{Mg} \mathrm{m}^{-3}$ ). The mixture was tuned using the rule of mixtures to give a density of about $0.860 \mathrm{Mg} \mathrm{m}^{-3}$. Spheroids of olive oil were added to the solution by pipetting $5 \mu \mathrm{L}$ droplets into the ethanol-water mixture to give spheres of about $2 \mathrm{~mm}$ diameter. The final density of the mixture was adjusted to the olive oil to give neutrally buoyant spheroids that act as tracer particles. The flow under varying fluid volumes $(60 \%$, $85 \%$, and $100 \%$ ) and rotation speeds $(5,10$, and $15 \mathrm{rpm})$ was investigated. The rotation speeds of 5,10 , and $15 \mathrm{rpm}$ correspond to velocities at the outer vessel wall of about 14,29, and $43 \mathrm{~mm} \mathrm{~s}^{-1}$ (55 mm diameter vessel). The motion of the olive oil tracer particles was recorded using a compact digital camera (Canon IXUS 510 HS). Recordings were made after the fluid rotation had reached a steady state to avoid any transient startup effects. The position and velocity of the fluid was calculated using a custom Matlab code. The user was prompted to track 4-5 of the oil tracer particles throughout the analysis period. From their known positions, the velocity vectors of the particles were calculated and plotted.

Figure 3 shows the experimental setup used to investigate the effect of on- and off-axis mounting of the reaction vessel on the fluid velocity fields. The reaction vessel (gray) was mounted away from the central axis of rotation. The rotation speed was $15 \mathrm{rpm}$, higher than the typical rotation speed the bioreactor would operate to establish whether centrifugal effects are significant.

For scaffold flow tracking, scaffolds $5 \mathrm{~mm}$ in diameter and $4 \mathrm{~mm}$ in height were used. The imaging vessel was filled with PBS, and a solitary scaffold was placed into the vessel. Scaffold imaging and tracking of the scaffold's midpoint was carried out, respectively, using the compact camera and Matlab code used for fluid flow analysis.

\section{Culture of fetal human osteoblasts}

Fetal human osteoblasts (fHObs) were purchased from Public Health England who obtain the cells from Cell Appli- cations, Inc. (San Diego, CA). Cells were cultured in McCoy's 5A medium without phenol red (Fisher Scientific), supplemented with $10 \%$ fetal bovine serum (Life Technologies), $1 \%$ Penicillin-Streptomycin (Life Technologies; 10,000 $\mathrm{U} \mathrm{mL}^{-1}$ ), and $30 \mu \mathrm{g} / \mathrm{mL}$ Vitamin $\mathrm{C}$ salt (L-Ascorbic acid, Insight Biotechnology) (complete medium) in a humidified atmosphere at $5 \% \mathrm{CO}_{2}$ and $37^{\circ} \mathrm{C}$. Cells were plated on T-75 flasks at $6.5 \times 10^{4}$ $\mathrm{cm}^{-2}$ and cultured until $85 \%$ confluent when they were passaged by trypsinization. fHObs were used at $\mathrm{p} 5$ for all experiments described in this study.

\section{Seeding of cells on CG scaffolds}

Scaffolds were rendered sterile for the purposes of shortterm cell culture by placing in $70 \%$ ethanol, washing thrice in PBS, and placing in UV light for $24 \mathrm{~h}$. Subsequently the scaffolds were soaked in complete medium for $24 \mathrm{~h}$ at $37^{\circ} \mathrm{C}$ before seeding. Prepared scaffolds were removed from medium, placed onto a sterile petri-dish, and excess medium removed by squeezing the scaffolds with sterile tweezers. The compressed scaffolds were then placed onto a sterile hydrophobic polytetrafluoroethylene (PTFE) membrane (Fisher Scientific) within a petri-dish. The scaffolds were seeded by placing a $50 \mu \mathrm{L}$ droplet on top of the scaffold containing $\sim 65,000$ cells. The compressed scaffold draws the cell suspension into itself, while the PTFE membrane prevents the suspension from flowing away so that it remains in contact with the scaffold. The cells were allowed to attach by incubation in a humidified incubator at $37^{\circ} \mathrm{C}$ for $4 \mathrm{~h}$. The scaffolds were then transferred to a 24-well plate and covered with $1 \mathrm{~mL}$ of culture medium.

\section{Determining cell proliferation in scaffolds}

Cell proliferation was evaluated by measuring cell number using the CyQUANT cell proliferation assay (Thermo Fisher Scientific). After cell culture, samples were flash frozen in liquid nitrogen and stored at $-80^{\circ} \mathrm{C}$ until analysis. Subsequently, samples were thawed and digested in $1 \mathrm{~mL}$ of papain digestion solution consisting of $1 \mathrm{mg} \mathrm{mL}^{-1}$ lyophilized papain (Sigma-Aldrich), $1.2 \mathrm{mg} \mathrm{mL}^{-1}$ L-cysteine (SigmaAldrich), and $5 \%(\mathrm{v} / \mathrm{v})$ of $20 \times$ CyQUANT lysis buffer in nuclease-free water (QIAGEN). Samples were digested at 

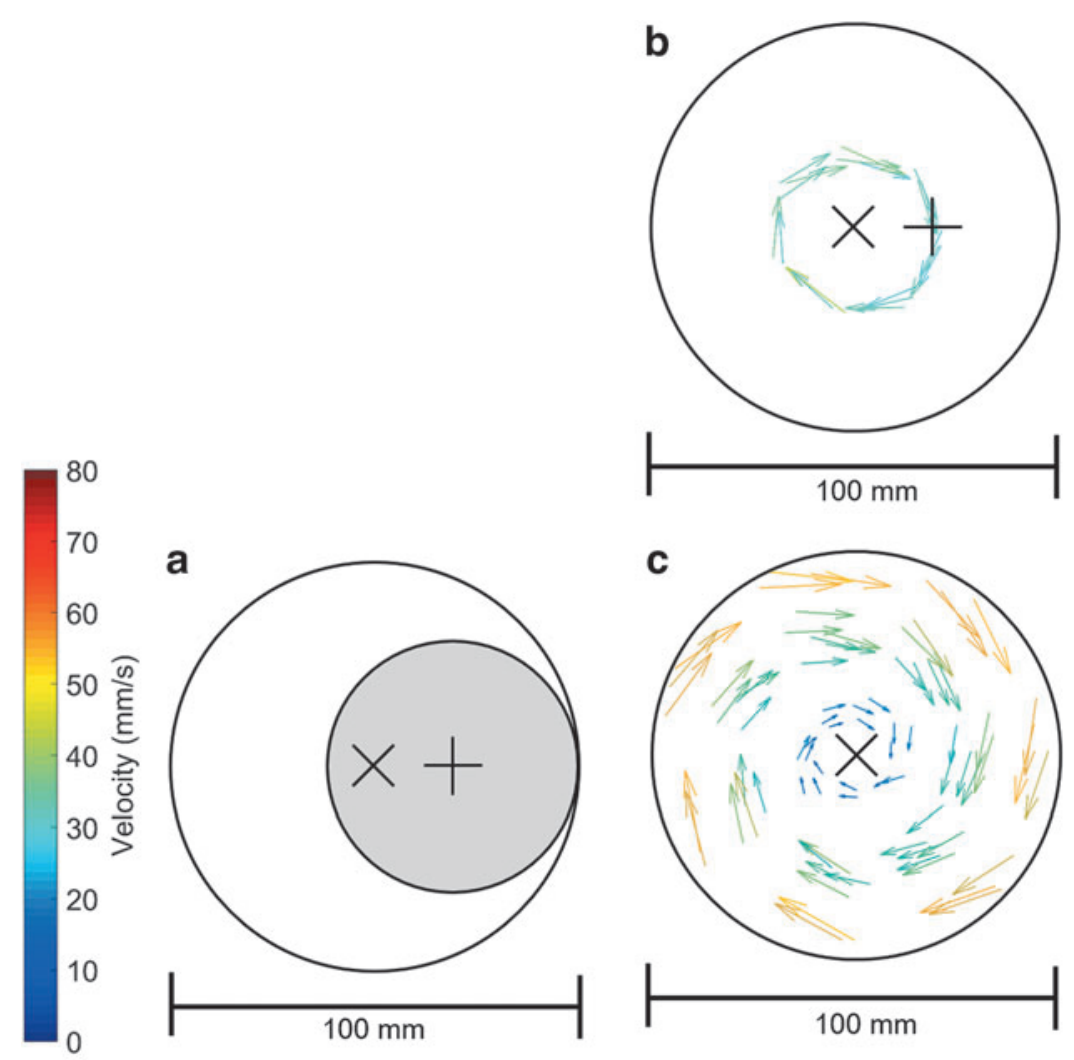

FIG. 3. Off-axis flow field analysis for a rotation speed of $15 \mathrm{rpm}$ showing (a) a schematic of the reaction vessel (gray) mounted off-axis and (b) the velocity of the center of the reaction vessel in the global coordinates. The fluid velocity field within the reaction vessel is shown in (c) the global and (d) local coordinate systems. ${ }^{\times}$Denotes the center of the global bioreactor rotation axis and ${ }^{+}$The center of the local rotation axis of the reaction vessel.

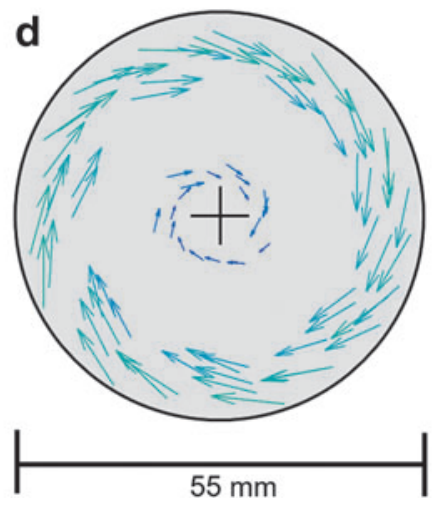

$60^{\circ} \mathrm{C}$ for $90 \mathrm{~min}$ before placing on an orbital shaker. The lysate was transferred in duplicate $100 \mu \mathrm{L}$ aliquots to a black 96-well plate to which $100 \mu \mathrm{L}$ CyQUANT GR dye solution $(200 \times$ dilution in papain digestion solution) was added. After incubating for $5 \mathrm{~min}$ at room temperature, the fluorescence was read on a spectrometer (FLUOstar OPTIMA, BMG Labtech) with an excitation and emission wavelength of 485/ $520 \mathrm{~nm}$. The signal was corrected using blank scaffold extracts treated in the same way and the signals converted to cell number using a standard curve prepared using known concentrations of fHObs.

\section{Imaging cell distribution in scaffolds}

The density of cells on the surface and in the center of the CG scaffolds following cell culture in the single and dual axis RWV bioreactors and under static conditions was evaluated by imaging cell nuclei stained with $4^{\prime} 6$-diamidino2-phenylindole (DAPI). Scaffolds were fixed in $4 \% \mathrm{w} / \mathrm{v}$ paraformaldehyde (Santa Cruz Biotechnology), rinsed twice in PBS, and cross-sectioned through the diameter of the cylindrical scaffold using a Feather ${ }^{\circledR} \mathrm{S} 35$ microtome blade (pfm medical). Cell nuclei were stained by the addition of 3 drops of Fluoroshield antifade mountant containing DAPI (Sigma) to the top surface and the cut surface of the sectioned scaffold and imaged in the center of each surface using a Zeiss Axio Observer Z1 fluorescent microscope.

\section{Optimization of an RWV bioreactor for cell culture}

The proliferation of fHObs, seeded as described above in CG scaffolds, was investigated when cultured as free floating scaffolds in the single axis RWV bioreactor. In this experiment the bioreactor chamber had a nominal fill volume of $85 \%$ complete medium. The effect of three speeds of rotation 5, 10, and $15 \mathrm{rpm}$ was studied, corresponding to velocities at the outer vessel wall of about 7,14 , and $21 \mathrm{~mm} \mathrm{~s}^{-1}(27 \mathrm{~mm}$ diameter vessel $-50 \mathrm{~mL}$ cell culture tubes). At 1, 7, 14, and 21 days, 
scaffolds were removed and cell number measured using the CyQUANT assay. After establishing that $10 \mathrm{rpm}$ produced optimum cell proliferation, the effect of varying the chamber fluid volume was studied while maintaining the rotation at $10 \mathrm{rpm}$. Three fill volumes were used as follows: $60 \%, 85 \%$, and $100 \%$ under two scaffold conditions, where the scaffolds were either free floating (unconstrained) or mounted in a fixed position along the long axis of the tube on needles that were held by a custom printed mount, which gripped the inside of the tube (constrained). Again, in both cases scaffolds were removed at 1,7, 14, and 21 days for CyQUANT assay. In each of these experiments proliferation was compared to that of cells in scaffolds cultured under static conditions. Two further experiments were performed comparing cells grown on unconstrained scaffolds in the single axis RWV bioreactor compared to scaffolds grown under static conditions or static and constrained conditions. Scaffolds were removed at several time points and cell number determined using the CyQUANT assay. In the second experiment scaffolds were removed at the final time point, sectioned through the middle of the scaffold, and the cell nuclei stained and imaged.

\section{Comparison of the effect of single and dual axis rotation on cell proliferation in scaffolds}

The proliferation of fHObs on CG scaffolds using dual axis rotation was compared to that in the single axis rotation bioreactor. Cells were seeded in the scaffolds as described, and 16 scaffolds were placed in each of the bioreactor chambers as either unconstrained or constrained scaffolds. In each bioreactor, the tubes had an $85 \%$ fill with complete medium, and the tubes were rotated at $10 \mathrm{rpm}$ in either a single axis or in both axes. At 1, 7, 14, and 21 days, scaffolds were removed and cell number measured using the $\mathrm{Cy}-$ QUANT assay. In these experiments cell proliferation was also determined in scaffolds kept under static conditions.

\section{Statistical analysis}

Quantitative evaluations were performed using triplicate samples per data point. Data are expressed as the arithmetic mean \pm standard deviation of these technical replicates. Each graph shows the results from an independent experiment. All analyses were carried out using IBM SPSS statistics
Fluid Velocity Field

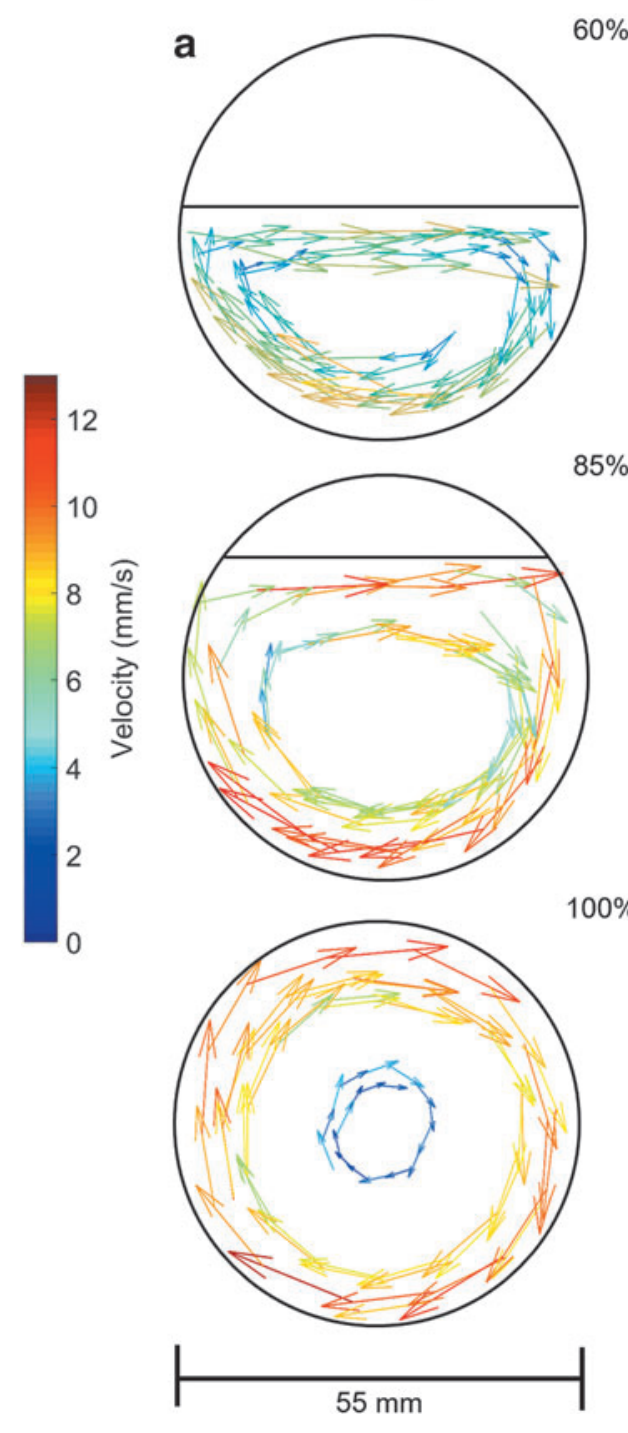

Track of Scaffold Mid-point

$\%$

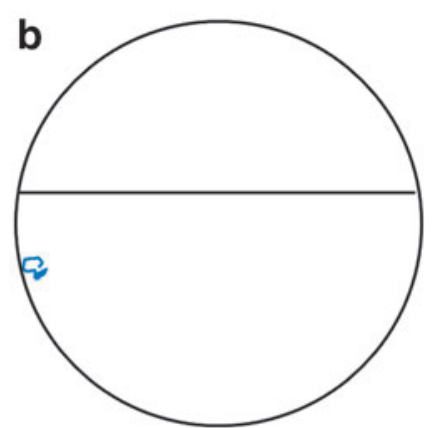

$85 \%$

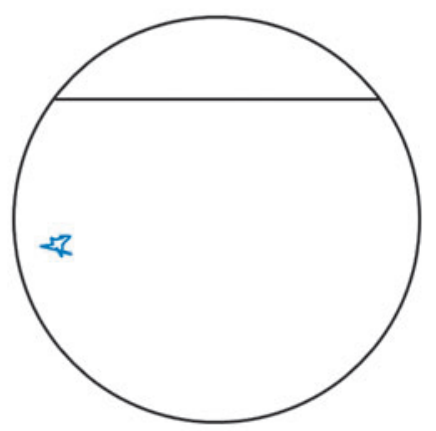

$100 \%$

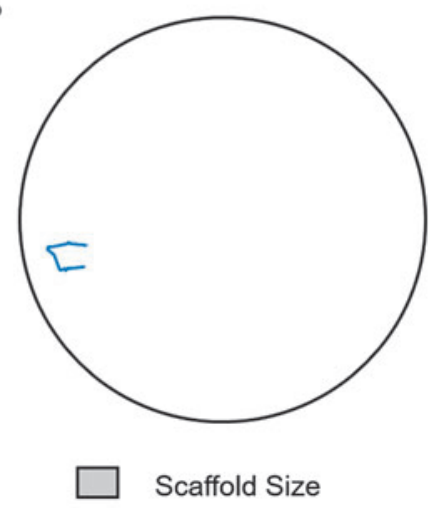

FIG. 4. (a) Flow velocity vector (b) scaffold motion at a rotation speed of $5 \mathrm{rpm}$ (outer vessel wall velocity of about $14 \mathrm{~mm}$ $\left.\mathrm{s}^{-1}\right)$, for different fluid to air ratios $(60 \%$, $85 \%$, and $100 \%$ ). The axis of rotation is orthogonal to gravity. 
version 23, and the statistical significance of differences in mean values within each experiment was evaluated using a two-way analysis of variance. Differences between pairs of means were described using Tukey's Honest Significant Difference post hoc test. A probability value of $95 \%(p<$ 0.05) was used to determine significance.

\section{Results}

Fluid flow and scaffold tracking-influence of fluid volume and rotation speed

The fluid velocity field when the rotation axis (and the long axis of the reaction vessels) is orthogonal to gravity is shown in Figures 4-6a at rotation speeds of 5, 10, and $15 \mathrm{rpm}$. Fluid to air ratios of $60 \%, 85 \%$, and $100 \%$ were considered. It can be seen that the fluid velocity vectors follow the shape of the liquid present. A decrease in velocity is observed as the fluid approaches the interface between the vessel, the fluid, and the air. The position of the scaffold's midpoint at the varying fluid volumes and rotation speeds is shown in Figures 4-6b. At a rotation speed of $5 \mathrm{rpm}$ (Fig. 4b), the scaffolds appear static at all three fluid ratios. At $10 \mathrm{rpm}$ (Fig. 5b), the scaffolds undergo periodic oscillations on the left side of the reaction vessel. At $15 \mathrm{rpm}$ (Fig. 6b), the scaffolds appear to trace out the streamlines of the fluid.

\section{Influence of rotation axis on fluid flow and scaffold motion}

When the rotation axis coincides with gravity (the long axis of the reaction vessel is still orthogonal to gravity), the fluid reaches perfect rigid body rotation at all rotation speeds as illustrated in Figure 7a. The scaffold's midpoint (Fig. 7b) traced out circular motion and aligned with the velocity vectors of the fluid at all speeds investigated. Figure 8 shows the experimentally measured velocity field for the reaction vessel center in the global (bioreactor) and local (vessel) coordinate systems. It can be seen that the fluid flow fields are equivalent if the reaction vessel is mounted on- or offaxis. Due to the relatively low speed of rotation, the fluid flow is dominated by the relative motion between reaction vessel wall and fluid.

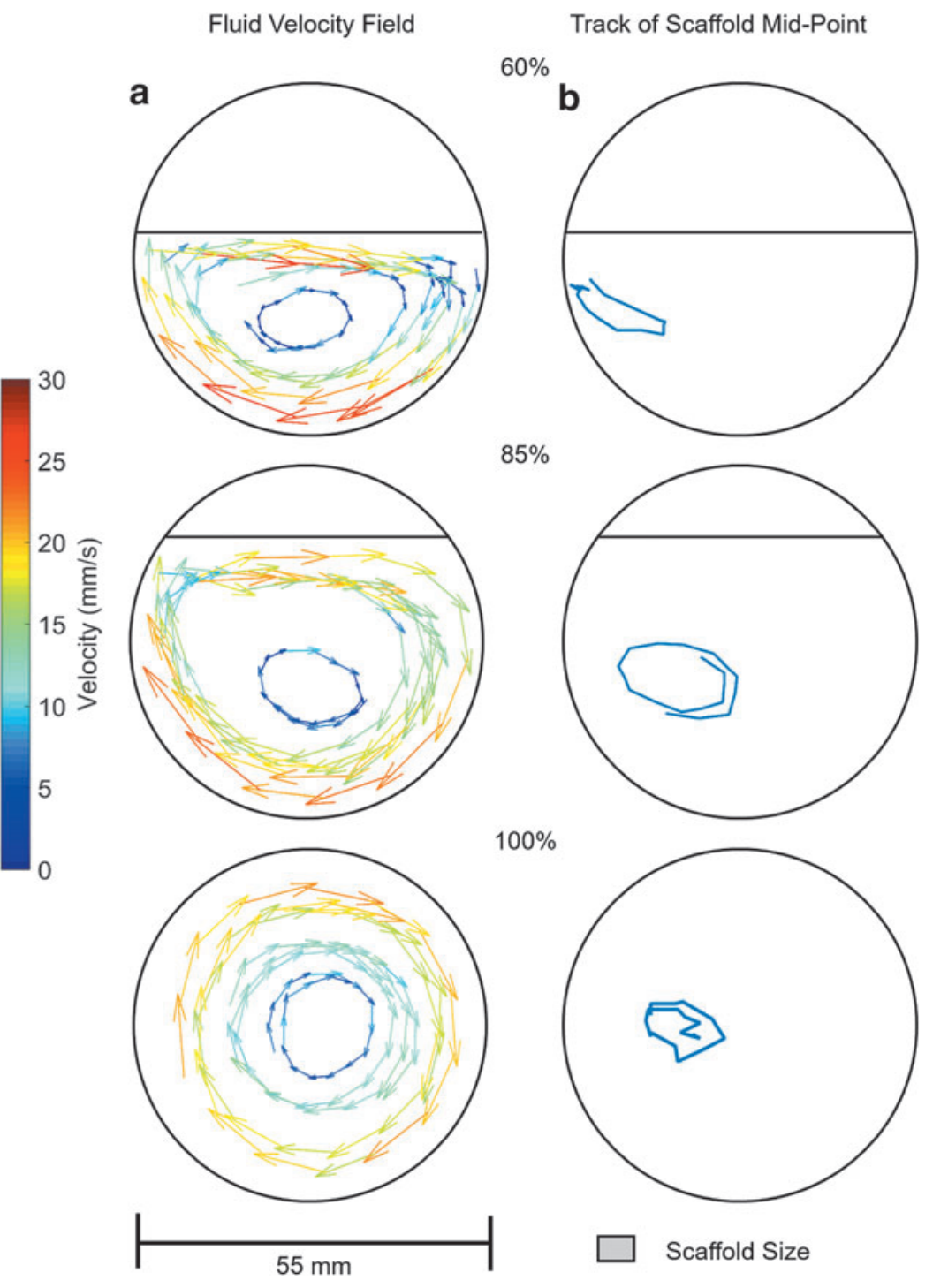

FIG. 5. (a) Flow velocity vector (b) scaffold motion at a rotation speed of $10 \mathrm{rpm}$ (outer vessel wall velocity of about $29 \mathrm{~mm}$ $\left.\mathrm{s}^{-1}\right)$, for different fluid to air ratios $(60 \%$, $85 \%$, and $100 \%$ ). The axis of rotation is orthogonal to gravity. 
Fluid Velocity Field

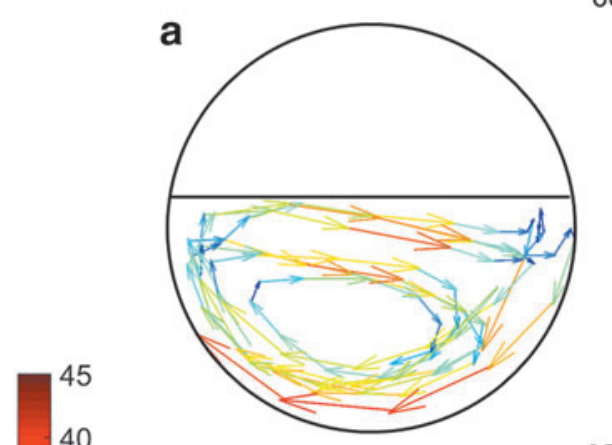

FIG. 6. (a) Flow velocity vector (b) scaffold motion at a rotation speed of $15 \mathrm{rpm}$ (outer vessel wall velocity of about $43 \mathrm{~mm}$ $\left.\mathrm{s}^{-1}\right)$, for different fluid to air ratios $(60 \%$, $85 \%$, and $100 \%)$. The axis of rotation is orthogonal to gravity.
40

35

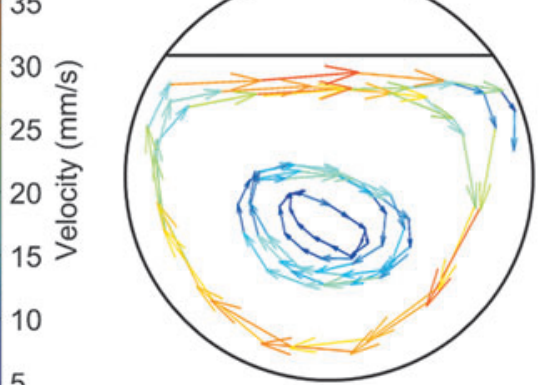

5

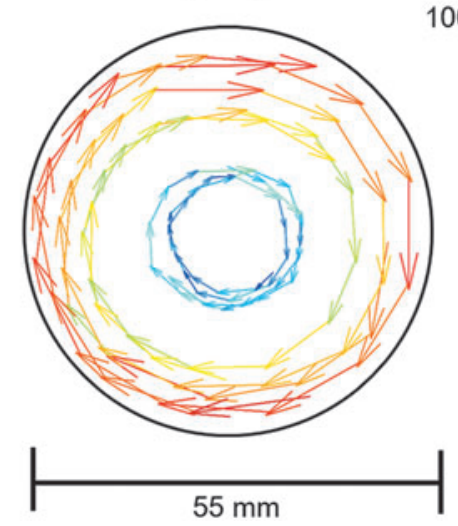

Track of Scaffold Mid-Point

$60 \%$

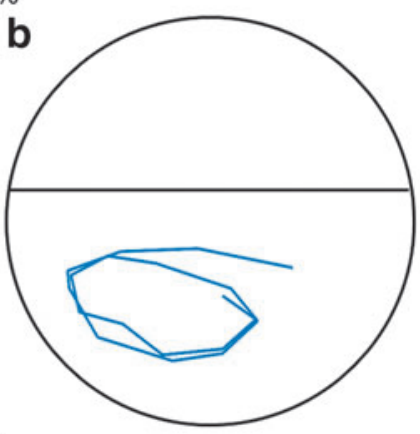

$85 \%$

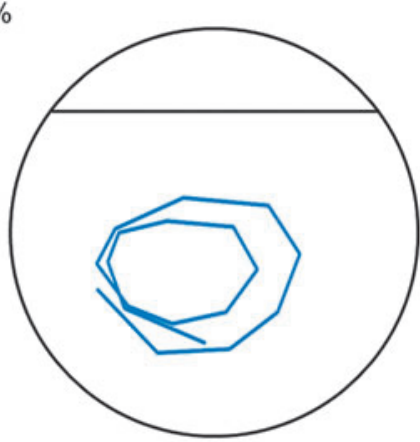

$00 \%$

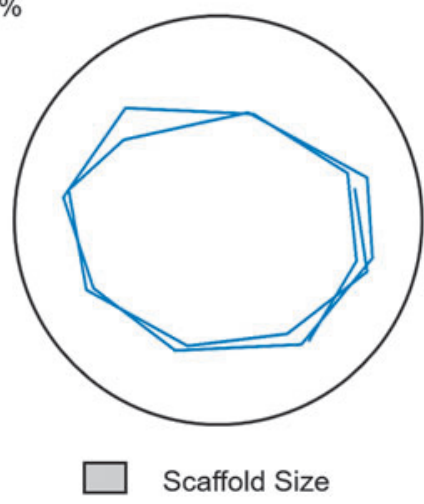

\section{Optimized bioreactor parameters for cell proliferation}

The cells in the unconstrained scaffolds increased in number from day 7 onward. When $10 \mathrm{rpm}$ was selected as the rotation speed and the percentage fill of the bioreactor tube was varied between $60 \%$ and $100 \%$, cell proliferation was significantly greater than in the static scaffolds at each time point from day 7 (Fig. 9a). In this study, the $85 \%$ fill volume produced significantly greater cell proliferation than $60 \%$ and $100 \%$ fill at days 14 and 21 . However, this was only seen when the scaffolds were unconstrained. When scaffolds were mounted on pins there was only a small nonsignificant increase in cell number with time, which was comparable to that seen in the static scaffolds for tubes that were $85 \%$ and $100 \%$ filled with medium (Fig. 9b). There was a dramatic significant decrease in cell number within the constrained scaffolds at $60 \%$ chamber fill. Cell proliferation was seen at each rotation speed tested in the $85 \%$ medium filled bioreactor, and the numbers were significantly greater than those in the static scaffolds at each time point (Fig. 9c). The increase was greatest at $10 \mathrm{rpm}$ where there were significantly more cells than at 5 or $15 \mathrm{rpm}$. Using further batches of cells in separate experiments, cell proliferation was significantly higher in the unconstrained scaffolds compared to static scaffolds (Fig. 10a) and both static and constrained scaffolds (Fig. 10b) after 15 days in the single axis RWV bioreactor. The distribution of cells on the scaffolds showed that while cells were present on the surface of all scaffolds (Fig. 10c-e), far more cells were present in the middle of the unconstrained scaffolds (Fig. 10f) than when scaffolds were constrained or cultured under static conditions (Fig. 10g, h).

\section{Comparison of single axis and dual axis rotation on cell proliferation in CG scaffolds}

The results of two independent replicate experiments comparing the proliferation of cells in the scaffolds cultured in the single axis RWV bioreactor and the biaxial rotating bioreactor are shown in Figure 11. The only difference in the two experiments is the absence of a constrained single 

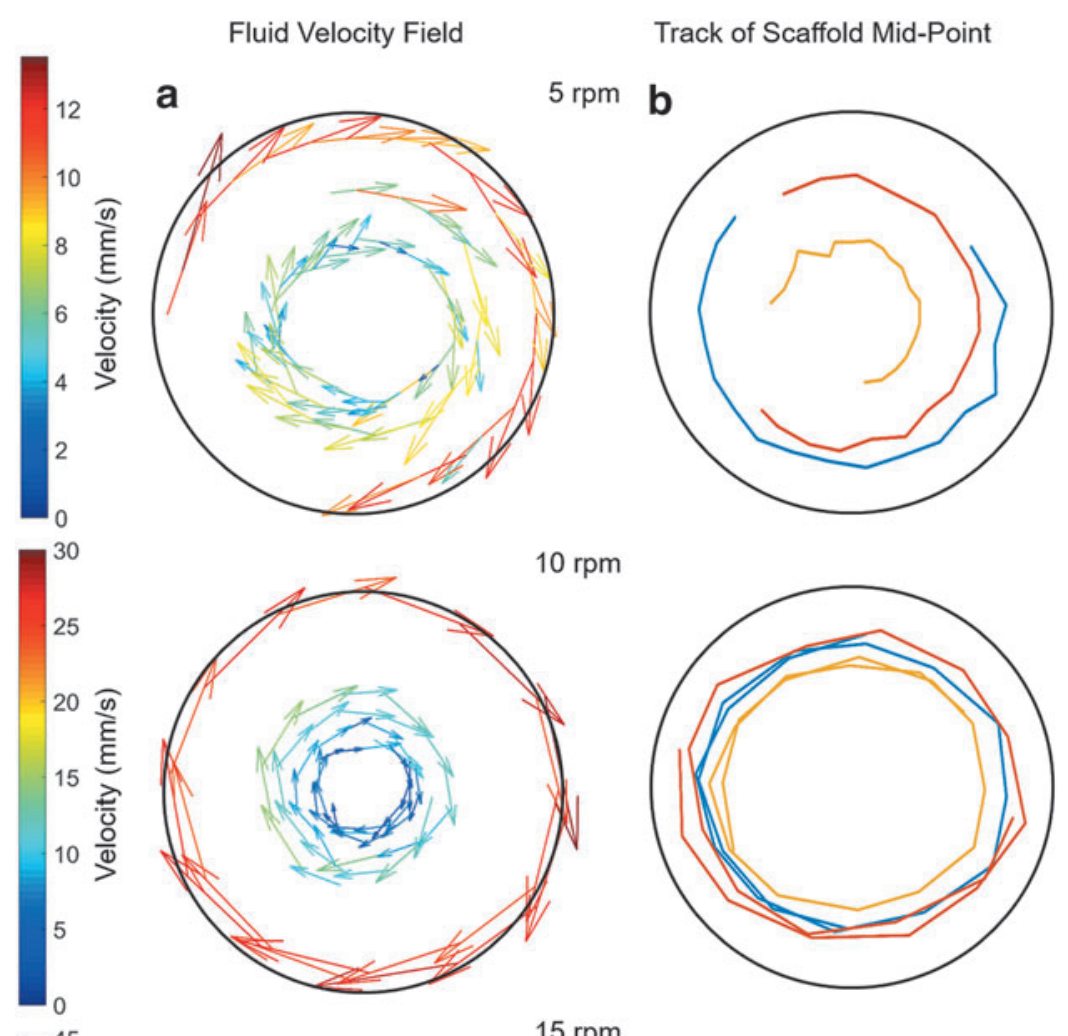

FIG. 7. (a) Flow velocity vector (b) scaffold motion at $100 \%$ fluid volume, for different rotation speeds $(5,10$, and $15 \mathrm{rpm})$. The axis of rotation is parallel to gravity.
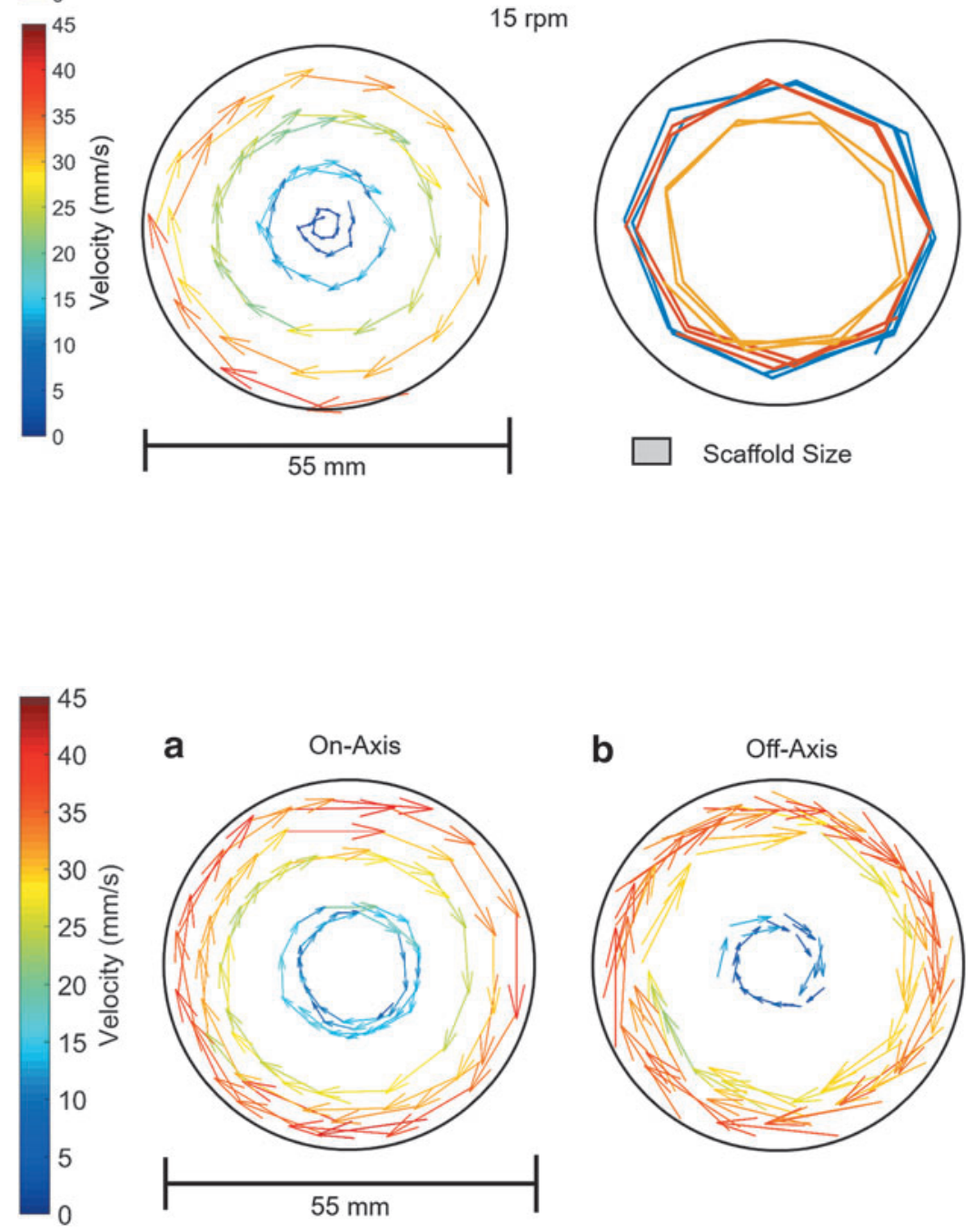

FIG. 8. Fluid velocity fields measured for a rotation speed of $15 \mathrm{rpm}$ (outer vessel wall velocity of about $43 \mathrm{~mm} \mathrm{~s}^{-1}$ ) when the reaction vessel is mounted (a) on-axis and (b) off-axis. 

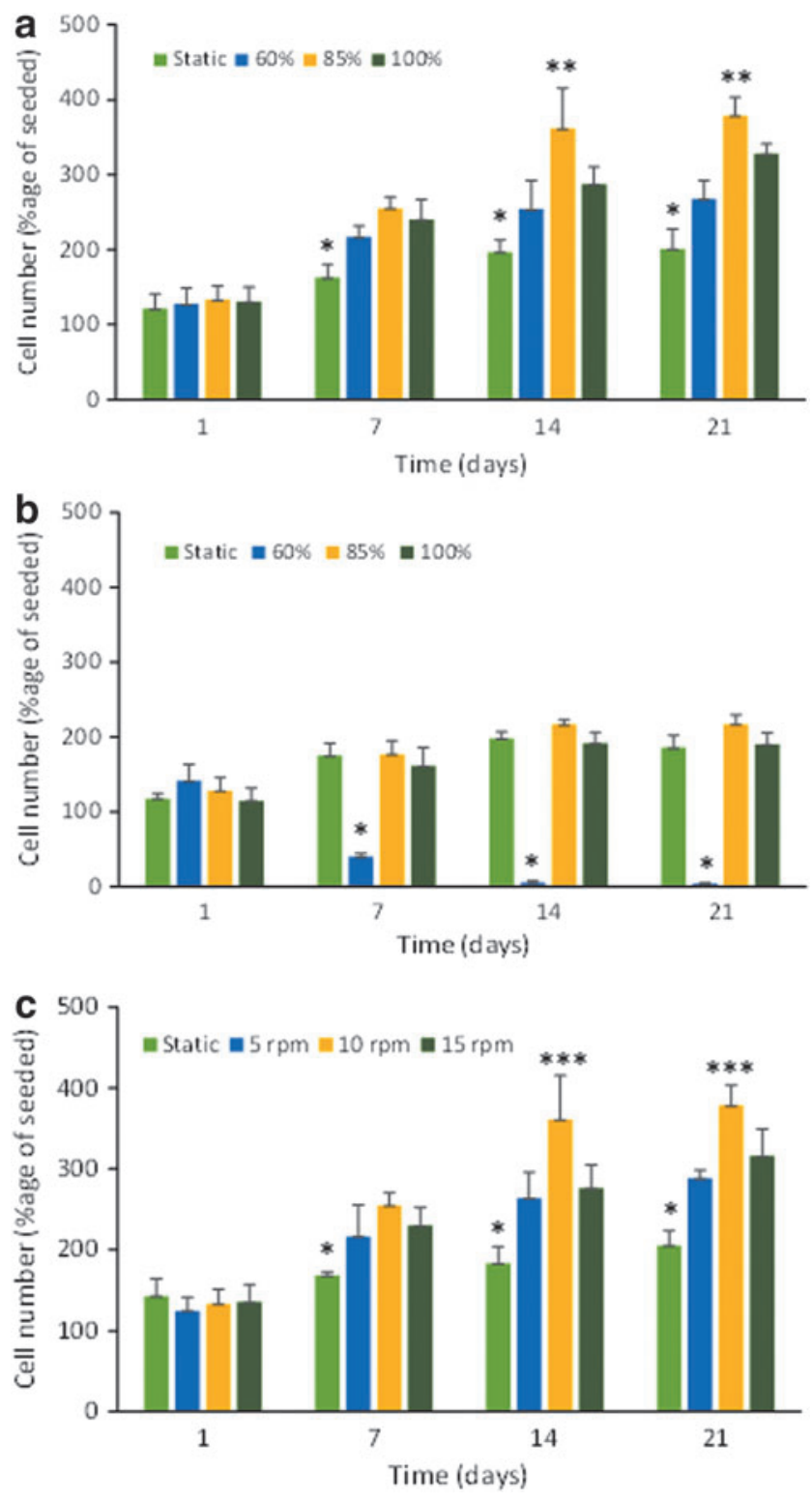

FIG. 9. Optimization of single axis bioreactor for cell culture. Cell number expressed as a percentage of cells seeded (mean $\pm \mathrm{SD} ; n=3$ technical replicates) for (a) a rotation speed of $10 \mathrm{rpm}$ while varying the chamber fill from $60 \%$ to $100 \%$ with unconstrained scaffolds, (b) a rotation speed of $10 \mathrm{rpm}$ while varying the chamber fill from $60 \%$ to $100 \%$ with fixed constrained scaffolds, and (c) an $85 \%$ full chamber while varying the rotation speed from 5,10 , and $15 \mathrm{rpm}$ (outer vessel wall velocities $\sim 7,14$, and $21 \mathrm{~mm} \mathrm{~s}^{-1}$, respectively) with unconstrained scaffolds. Each experiment also includes an $85 \%$ fill static control group. For each graph, $* p<0.002$ compared to the other groups at that time point, $* * p<0.01$ compared to $60 \%$ and $100 \%$ fluid volume, and $* * * p<0.002$ compared to 5 and $15 \mathrm{rpm}$. SD, standard deviation.

axis scaffold group in the first experiment. There was good agreement between the results with significantly greater proliferation in both the single axis and dual axis rotation bioreactors compared to the static control groups after 14 and 21 days, but no significant proliferation in the con- strained groups. However, there was no significant difference between the cell number after culture under single axis rotation and culture with biaxial rotation at any time point. The number of cells on the scaffold surface appeared similar between unconstrained scaffolds cultured under single axis and dual axis rotation (Fig. 11c, d). The number of cells in the center of these two scaffolds while fewer than on the surface also appeared similar between the two scaffolds (Fig. 11f, g). Fewer cells were seen on both the surface (Fig. 11e) and in the middle (Fig. 11h) of the static scaffold compared to the corresponding position for the unconstrained scaffolds cultured in both bioreactors.

\section{Discussion}

The role of combining two axes of rotation on fluid flow and cell growth in a dual axis RWV bioreactor is not clear despite the demonstration of improved cell growth in a commercialized biaxial bioreactor. ${ }^{10,11}$ Potential benefit could be due to improved mass transport into the cell/ scaffold construct thereby removing the deficit in medium and dissolved gas transfer in and out of the scaffold; in addition, the improved medium flow would increase shear forces on the cells in the scaffold, which can increase cell proliferation. ${ }^{16,17}$ We developed simple low-cost single and dual axis RWV bioreactors to allow a comparison of the proliferation of cells. We used CG scaffolds for culture of fHObs in the bioreactors as these scaffolds have been well characterized $^{15,18}$ and support bone cell culture. ${ }^{19,20}$ They also have a suitable density for unconstrained culture in RWV bioreactors without the use of high rotational speeds.

To understand the effect of rotation speed and chamber fill volume on fluid velocity and direction and unconstrained scaffold movement, fluid flow analysis and scaffold tracking were conducted and the conditions for cell culture were optimized in the single axis RWV. Rotating wall bioreactors have traditionally consisted of reaction vessels mounted on-axis. However, this is not a design requirement. As illustrated in Figure 8, off- and on-axis mounting of the reaction vessels provides equivalent relative flow fields at the rotation speeds used in this study. Hence the fluid flow fields when multiple reaction vessels are mounted off-axis, as in the bioreactors developed in this study, are expected to be equivalent to that of a single reaction vessel mounted on-axis.

Of particular interest in this study was the effect of different ratios of fluid to air in the bioreactor tubes. One limitation in reaction vessel design is having sufficient area for gas exchange. With a bioreactor vessel completely filled with culture medium, this requires a sufficiently large gas permeable window. A novel approach to bioreactor vessel design used "off the shelf" tubes, in which a window with a gas permeable silicone membrane was inserted. ${ }^{21}$ This approach provided the idea for the bioreactor vessels used in this study, but they were simplified further by having the area for gas exchange provided by holes in the lid as found in static cell culture flasks. This gives a relatively small window and sufficient gas exchange is provided by having both fluid and air within the reaction vessel, which greatly increases the surface area for gas exchange. In this work, the flow field was measured for fluid volumes of $60 \%, 85 \%$, and $100 \%$ at a range of rotation speeds as shown in Figures 4-6 

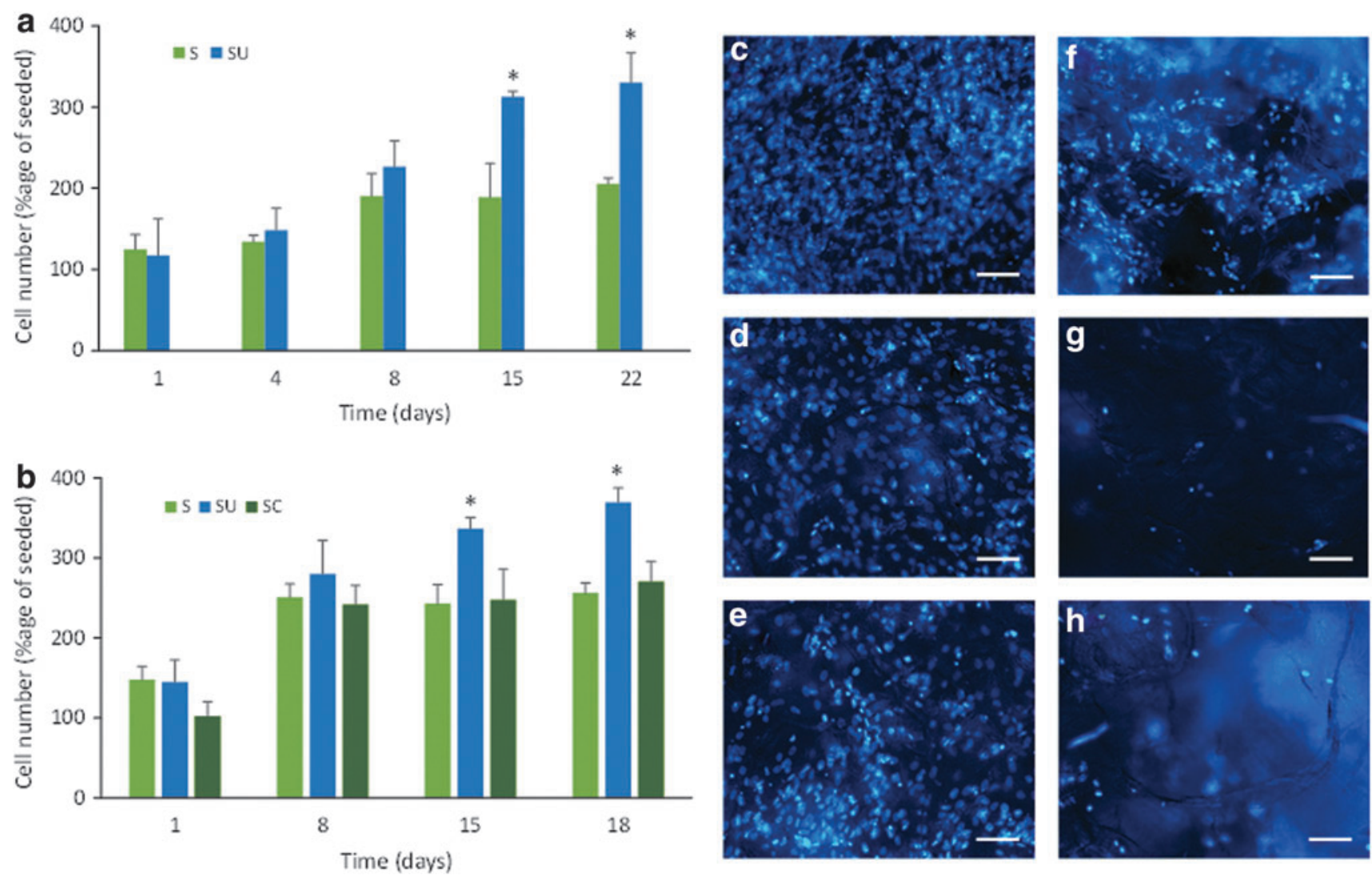

FIG. 10. Proliferation of cells grown under optimized conditions (10 rpm and 85\% chamber fill) for two experiments showing significantly greater cell number $(* p<0.01)$ as a percentage of cells seeded (mean $\pm \mathrm{SD} ; n=3$ technical replicates) from 15 days on unconstrained (SU) scaffolds compared to static (S) (a) and to both static and constrained (SC) scaffolds (b). Images taken at day 18 show abundant cells on the surface of scaffolds grown under single axis, unconstrained (c), single axis, constrained (d), and static (e) conditions but only in the center of unconstrained (f) and not constrained (g) or static (h) scaffolds. Scale bar $=50 \mu \mathrm{m}$.

(Fluid volumes below $60 \%$ resulted in turbulent flow). At all rotation speeds, the fluid vectors tracked the volume the fluid occupied. Below 100\% full, a decrease in velocity was observed as the fluid approached the reaction vessel, fluid, and air interface. This decreasing velocity is expected due to conservation of momentum. Fluid on the free surface has to cover a shorter distance than it would if the reaction vessel was $100 \%$ filled with fluid, hence resulting in a lower velocity.

The effect of fill volume on cell proliferation clearly showed that at $10 \mathrm{rpm}$ (outer vessel wall velocity $\sim 14 \mathrm{~mm}$ $\mathrm{s}^{-1}$ in a $27 \mathrm{~mm}$ diameter vessel) and with unconstrained scaffolds, $85 \%$ fill was optimum and this was also found at $5 \mathrm{rpm}$ and $15 \mathrm{rpm}$ (results not shown). A lower cell number after culture with $100 \%$ chamber fill is likely due to a reduced surface for gas exchange, whereas with $60 \%$ fill, the flow analysis indicates a lower fluid velocity which is unable to support the scaffold in free fall leading to collisions with the vessel wall alongside reduced mass transfer. When the scaffolds were constrained in the bioreactor vessel, cell proliferation did not benefit from vessel rotation. The fluid flow studies showed that the scaffolds would be moving at the same velocity and direction of the fluid, hence they would be limited if there is any net mass transfer of fluid into the scaffold. The scaffolds are hence effectively exist- ing in similar conditions to static culture and hence giving equivalent proliferative performance. With $60 \%$ fluid fill, the resulting reduction in cell number was due to the scaffolds having to pass through the air gap during rotation. This resulted in bubble formation and loss of cell viability.

The speed of rotation had important effects on the unconstrained scaffolds. At the lowest rotation speed of $5 \mathrm{rpm}$ (outer vessel wall velocity $\sim 14 \mathrm{~mm} \mathrm{~s}^{-1}$ in a $55 \mathrm{~mm}$ diameter vessel), the scaffold appeared static when viewed externally at all fluid ratios (Fig. 12a). This type of scaffold motion is known as suspension and occurs when the lift force due to the fluid flow is equal to that exerted by gravity. This occurs at the terminal velocity of the scaffold (the scaffold has zero acceleration). At $10 \mathrm{rpm}$ (outer vessel wall velocity $\sim 29 \mathrm{~mm} \mathrm{~s}^{-1}$ ), the scaffold undergoes periodic oscillations on the left side of the vessel (Fig. 12b). This occurs when the fluid flow provides sufficient lift force to accelerate the scaffold against gravity and causes the scaffold to be transported with the flow in the left region of the reaction vessel, close to the reaction vessel wall. As the direction of flow changes from vertical to horizontal toward the upper part of the reaction vessel, there is no longer sufficient vertical lift force from the fluid to overcome gravity. The result of this is that the scaffold starts to descend under gravity through the fluid toward the center of 
a
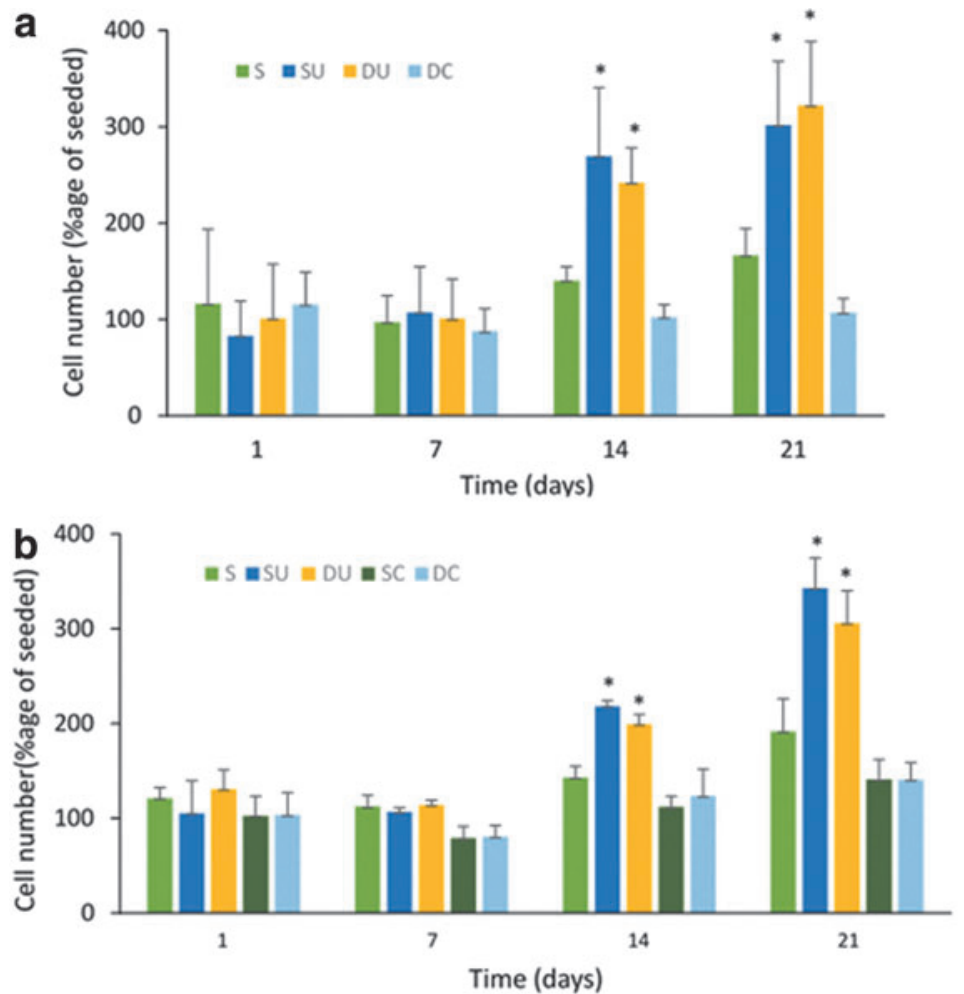
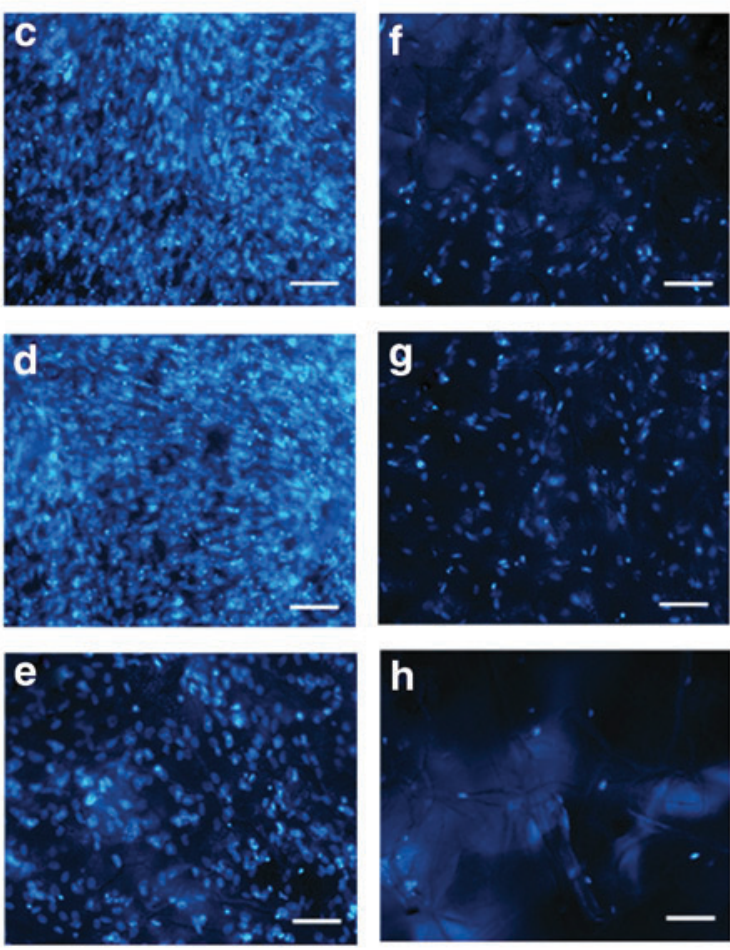

FIG. 11. Results of two replicate experiments, (a) and (b), each showing cell number expressed as a percentage of cells seeded (mean $\pm \mathrm{SD} ; n=3$ technical replicates) when cultured under static $(\mathrm{S})$, single axis rotation, unconstrained (SU), dual axis rotation, unconstrained (DU), and dual axis rotation, constrained (DC). In addition single axis rotation, constrained (SC) is shown in (b). ${ }^{*} p<0.05$ compared to static. Corresponding images are of cell nuclei stained at D21 showing numerous cells on the surface of both SU and DU scaffolds (c, d) with fewer, but still considerable numbers of cells, in the center (f, g). Less cells were seen on the surface (e) and center (h) of the static scaffold compared to their counterparts cultured under both single and dual axis rotation. Scale bar $=50 \mu \mathrm{m}$.

the reaction vessel. As the scaffold starts to approach the bottom of the reaction vessel, it encounters higher speed fluid flow that provides sufficient lift force to once again overcome gravity and accelerate the scaffold to the same velocity of the flow, repeating the motion. This motion has been previously described. ${ }^{9,13,14}$ At $15 \mathrm{rpm}$ (outer vessel wall velocity $\sim 43 \mathrm{~mm} \mathrm{~s}^{-1}$ ), the force exerted by the fluid is sufficient to accelerate the scaffold into steady orbital motion of the bioreactor (Fig. 12c). The force due to the fluid motion accelerates the scaffold to sufficient velocity that the acceleration due to gravity has a reduced influence on the scaffold motion. The lift force due to fluid flow is zero as the scaffold moves approximately aligned with the fluid field, with limited relative difference in velocity between scaffold and fluid. The circular motion of the scaffold is maintained by the pressure field of the fluid within the reaction vessel.

These regimes of scaffold motion have been previously described for scaffolds rotating in $100 \%$ full reaction vessels, ${ }^{13,14}$ but not when the fluid ratio is reduced. Depending on the fluid volume, the flow velocity vectors were varied slightly, and hence, the scaffold flow path was altered. This was best observed at $15 \mathrm{rpm}$ (Fig. 5b), where at lower fluid volumes, the scaffold flow path was more elliptical compared to the $100 \%$ fluid filled reaction vessel. At $60 \%$ fluid volume, the region where the velocity vectors are primarily in a vertical direction was reduced so the scaffold motion was constrained to the lower region of the reaction vessel.
At a rotation speed of $5 \mathrm{rpm}$ and $60 \%$ fluid volume, the force due to the fluid was never sufficient to overcome gravity, and hence, the scaffold was observed rolling along the reaction vessel wall as shown in Figure $4 \mathrm{~b}$. When the volume of fluid reached $85 \%$, the fluid had sufficient velocity and, hence, forced to equal gravity and to undergo suspension.

To increase the transfer of nutrients into the scaffold, the optimum scaffold motion is suspension as in this regime the largest difference between fluid and scaffold velocities is observed. In both periodic oscillation and orbital motion, the scaffold has a component of velocity aligned with the fluid motion as observed in Figures 5 and 6, resulting in a reduction in the net transfer of fluid into the scaffold. In orbital motion, the scaffold moves at the same speed and on the same streamlines as the fluid, hence the net fluid transfer into the scaffold is approximately zero. The oscillatory motion does have periods of the motion where the fluid flow and scaffold motion are different as observed in Figure 5, hence allowing mass transfer. However, this is only for part of the scaffold motion so the result is still reduced mass transfer of fluid into the scaffold compared to suspension motion. From this it is predicted that having the scaffold in continuous suspension (free fall, outer vessel wall velocity $\leq 14 \mathrm{~mm} \mathrm{~s}^{-1}$ ) would give optimum mass transfer of fluid and hence best conditions for culture.

When translated to the cell culture tubes used as vessels in the bioreactors, a rotational speed of $10 \mathrm{rpm}$ provides an 

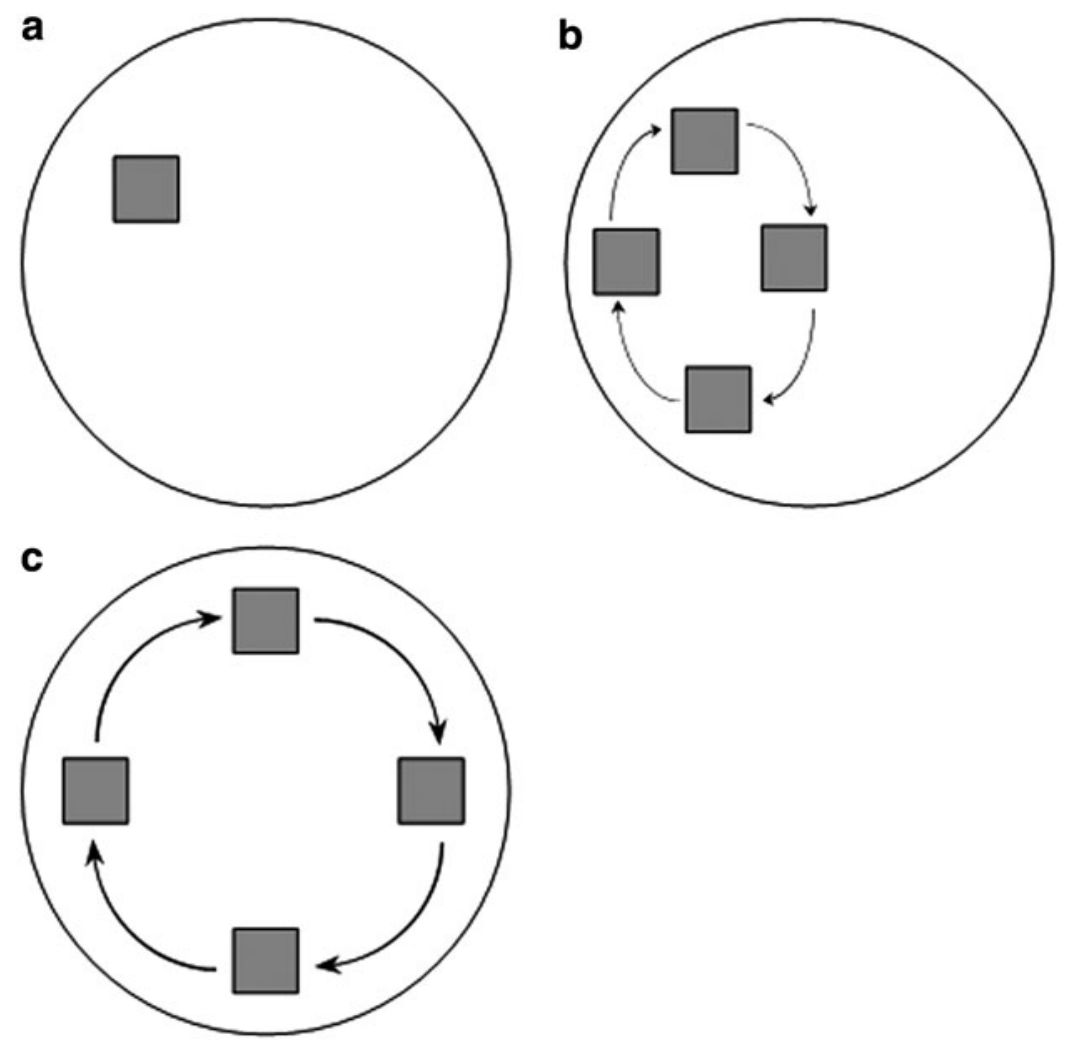

equivalent outer vessel wall velocity of $\sim 14 \mathrm{~mm} \mathrm{~s}^{-1}$ to that observed in the particle tracking experiments, and the cell culture results support the above prediction with $10 \mathrm{rpm}$ producing the highest cell number in the scaffolds after 14 and 21 days of culture. At $15 \mathrm{rpm}\left(\sim 29 \mathrm{~mm} \mathrm{~s}^{-1}\right)$ there would have been periodic oscillation resulting in reduced mass transfer and this produced the lower cell proliferation seen. At $5 \mathrm{rpm}$ there would be insufficient flow to raise the scaffold to terminal velocity producing suspension, resulting in reduced cell proliferation. The assumption that increased mass transport of oxygen and nutrients into the scaffold, greater shear forces on the cells present, or both of these are important for the increased cell proliferation seen at $10 \mathrm{rpm}$, and $85 \%$ chamber fill was supported by microscopy showing greater cell density in the center of the unconstrained scaffolds cultured in the single axis RWV bioreactor compared to static and constrained scaffolds. The high density of cells on the surface of the scaffolds indicates that differences in cell proliferation could also occur here.

The different scaffold motion regimes seen in this study only apply when the rotation axis is orthogonal to gravity. When the axis of rotation is aligned with gravity, the scaffold undergoes perfect rigid body rotation at all speeds as observed in Figure 6. This would be expected as in this plane of rotation the only forces acting on the scaffold are surface forces due to fluid motion. This suggests that the addition of a second axis of rotation, assuming that fluid motion due to each axis of rotation superimposes, would not give any improvements to the mass transfer into a scaffold compared to single axis rotation. The cell culture results provide evidence that this was indeed the case as the replicate experiments showed no significant differences in cell number on the scaffolds at 14 and 21 days between single and dual axis rotation with both unconstrained scaffolds (both replicates) and constrained scaffolds (single experiment only). There was however significantly increased proliferation of cells in the unconstrained scaffolds for both the single axis and dual axis bioreactor compared to cells grown in static scaffolds. Cell imaging also showed a similar density of cells in the center of the unconstrained scaffolds cultured under both single axis and dual axis rotation. In both cases this was greatly increased compared to cell numbers in the center of scaffolds cultured under static conditions. Cell proliferation on the surface of the scaffold will have contributed to the higher cell number seen on the unconstrained scaffolds; however, these results provide further evidence that under optimal rotation and chamber fill conditions there was increased mass transport supporting cells at the center of these scaffolds. The results confirm the advantage of RWV bioreactor culture seen in the optimization experiments when the rotation is $10 \mathrm{rpm}$ (free-fall state) in a vessel $85 \%$ filled with culture medium. The absence of any improvement in cell proliferation in constrained scaffolds seen in this study under dual axis rotation contrasts with the benefits previously reported for biaxial rotation of constrained scaffolds. ${ }^{10,11}$ Differences in the bioreactor configuration include that axes of rotation in the previous studies ${ }^{10,11}$ were orthogonal to gravity, whereas in the study reported here the second axis was parallel to gravity. However, fluid flow and scaffold motion are expected to be equivalent if the scaffolds are constrained. A further difference between the two systems is that the biaxial bioreactor in the previous studies included a perfusion loop, so it is possible that the biaxial motion of scaffolds through an independent fluid field gave the observed improvements in mass transfer and hence culture performance. 


\section{Conclusions}

In this study, the effect of rotation on fluid flow and unconstrained scaffold movement in a RWV bioreactor has been evaluated. Low cost single and dual axis rotating bioreactors were developed to investigate the benefit of dual axis rotation on cell proliferation in both free floating and constrained scaffolds. The results provide a theoretical basis for the lack of effect of dual axis rotation seen in the cell culture experiments. It is likely that further improvements to the culture of cells in these systems would require the addition of perfusion.

\section{Acknowledgments}

This research was supported by the European Research Council (Grant No. 240446) and the EPSRC (EP/E025862/ 1). Financial support for M.C.V. and R.A.B. has been provided through the WD Armstrong studentship and the National Institute for Health Research, respectively.

\section{Disclosure Statement}

No competing financial interests exist.

\section{References}

1. Hubble, M.J.W. Bone grafts. Surg Technol Int 10, 261, 2002.

2. Sladkova, M., and de Peppo, G. Bioreactor systems for human bone tissue engineering. Processes 2, 494, 2014.

3. Haycock, J.W. 3D cell culture: a review of current approaches and techniques. In: Haycock, J.W., ed. 3D Cell Culture: Methods and Protocols. Volume 695 of the series Methods in Molecular Biology. Humana Press, eBook (Springer Protocols), 2011, p. 1.

4. Diederichs, S., Roker, S., Marten, D., Peterbauer, A., Scheper, T., van Griensven, M., and Kasper, C. Dynamic cultivation of human mesenchymal stem cells in a rotating bed bioreactor system based on the $\mathrm{Z}(\mathrm{R}) \mathrm{RP}$ platform. Biotechnol Prog 25, 1762, 2009.

5. Song, K.D., Liu, T.Q., Cui, Z.F., Li, X.Q., and Ma, X.H. Three-dimensional fabrication of engineered bone with human bio-derived bone scaffolds in a rotating wall vessel bioreactor. J Biomed Mater Res A 86, 323, 2008.

6. Song, K.D., Wang, H., Zhang, B.W., Lim, M., Liu, Y.C., and Liu, T.Q. Numerical simulation of fluid field and in vitro three-dimensional fabrication of tissue-engineered bones in a rotating bioreactor and in vivo implantation for repairing segmental bone defects. Cell Stress Chaperones 18, 193, 2013.

7. Song, K.D., Yang, Z.M., Liu, T.Q., Zhi, W., Li, X.Q., Deng, L., and Ma, X.H. Fabrication and detection of tissueengineered bones with bio-derived scaffolds in a rotating bioreactor. Biotechnol Appl Biochem 45, 65, 2006.

8. Zhu, X.H., Arifin, D.Y., Khoo, B.H., Hua, J.S., and Wang, C.H. Study of cell seeding on porous poly(D,L-lactic-coglycolic acid) sponge and growth in a Couette-Taylor bioreactor. Chem Eng Sci 65, 2108, 2010.

9. Botchwey, E.A., Pollack, S.R., Levine, E.M., and Laurencin, C.T. Bone tissue engineering in a rotating bioreactor using a microcarrier matrix system. J Biomed Mater Res 55, 242, 2001.
10. Zhang, Z.Y., Teoh, S.H., Chong, W.S., Foo, T.T., Chng, Y.C., Choolani, M., and Chan, J. A biaxial rotating bioreactor for the culture of fetal mesenchymal stem cells for bone tissue engineering. Biomaterials 30, 2694, 2009.

11. Zhang, Z.Y., Teoh, S.H., Teo, E.Y., Chong, M.S.K., Shin, C.W., Tien, F.T., Choolani M.A., and Chan, J.K.Y. A comparison of bioreactors for culture of fetal mesenchymal stem cells for bone tissue engineering. Biomaterials 31, 8684, 2010.

12. Singh, H., Teoh, S.H., Low, H.T., and Hutmacher, D.W. Flow modelling within a scaffold under the influence of uni-axial and bi-axial bioreactor rotation. J Biotechnol 119, 181,2005

13. Cummings, L.J., Sawyer, N.B.E., Morgan, S.P., Rose, F., and Waters, S.L. Tracking large solid constructs suspended in a rotating bioreactor: a combined experimental and theoretical study. Biotechnol Bioeng 104, 1224, 2009.

14. Liu, T.Q., Li, X.Q., Sun, X.Y., Ma, X.H., and Cui, Z.F. Analysis on forces and movement of cultivated particles in a rotating wall vessel bioreactor. Biochem Engin J 18, 97, 2004.

15. Varley, M.C., Neelakantan, S., Clyne, T.W., Dean, J., Brooks, R.A., and Markaki, A.E. Cell structure, stiffness and permeability of freeze-dried collagen scaffolds in dry and hydrated states. Acta Biomaterialia 33, 166, 2016.

16. Cartmell, S.H., Porter, B.D., Garcia, A.J., and Guldberg, R.E. Effects of medium perfusion rate on cell-seeded threedimensional bone constructs in vitro. Tissue Engin 9, 1197, 2003.

17. Kapur, S., Baylink, D.J., and Lau, K.H.W. Fluid flow shear stress stimulates human osteoblast proliferation and differentiation through multiple interacting and competing signal transduction pathways. Bone 32, 241, 2003.

18. Harley, B.A., Leung, J.H., Silva, E., and Gibson, L.J. Mechanical characterization of collagen-glycosaminoglycan scaffolds. Acta Biomaterialia 3, 463, 2007.

19. Murphy, C.M., Haugh, M.G., and O'Brien, F.J. The effect of mean pore size on cell attachment, proliferation and migration in collagen-glycosaminoglycan scaffolds for bone tissue engineering. Biomaterials 31, 461, 2010.

20. Tierney, C.M., Jaasma, M.J., and O'Brien, F.J. Osteoblast activity on collagen-GAG scaffolds is affected by collagen and GAG concentrations. J Biomed Mater Res A 91, 92, 2009.

21. Terai, H., Hannouche, D., Ochoa, E., Yamano, Y., and Vacanti, J.P. In vitro engineering of bone using a rotational oxygen-permeable bioreactor system. Mat Sci Eng C 20, 3, 2002.

Address correspondence to: Roger A. Brooks, PhD

Division of Trauma and Orthopaedic Surgery Cambridge University Box 180 Addenbrooke's Hospital Hills Road Cambridge CB2 OQQ United Kingdom

E-mail:rb10003@cam.ac.uk

Received: September 2, 2016

Accepted: November 26, 2016

Online Publication Date: February 22, 2017 